\title{
CATÁLOGO DE ERRORES ASOCIADOS AL APRENDIZAJE DE LA TEORIA DE CONJUNTOS
}

\section{Mariela Lilibeth Herrera y Celsa Álvarez}

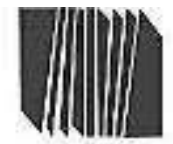

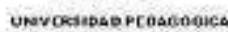

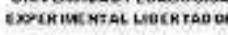
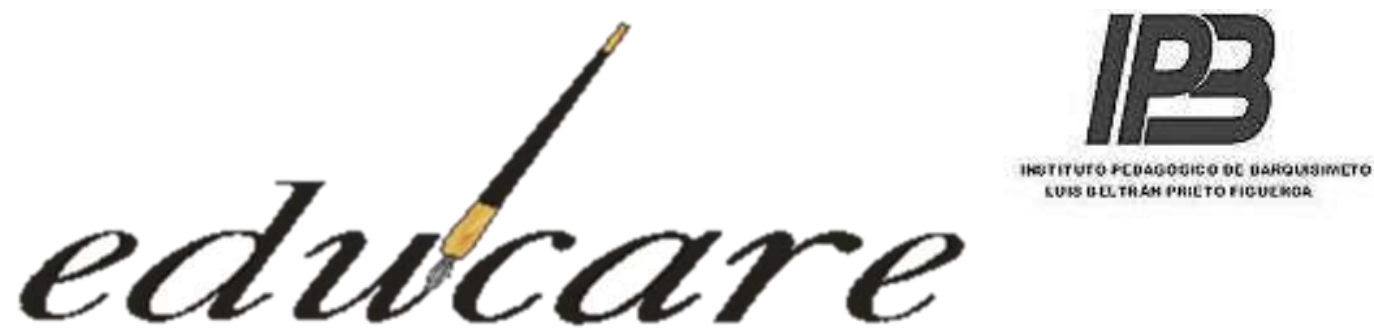

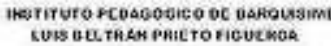

Órgano Divulgativo de la Subdirección de Investigación y Postgrado del Instituto Pedagógico de Barquisimeto "Lıis Beltrán Prieto

Figueroa"

\section{Volumen 22 № 3}

Septiembre - Diciembre 2018

CATÁLOGO DE ERRORES ASOCIADOS AL APRENDIZAJE DE LA TEORIA DE CONJUNTOS

CATALOG OF ERRORS ASSOCIATED WITH THE LEARNING OF THE SETS THEORY

\section{S N $: 2244-7296$}

\author{
Autor \\ Mariela Lilibeth Herrera \\ Celsa Álvarez \\ Universidad de Carabobo \\ Venezuela
}




\section{CATÁLOGO DE ERRORES ASOCIADOS AL APRENDIZAJE DE LA TEORIA DE CONJUNTOS}

Mariela Lilibeth Herrera y Celsa Álvarez

Pp 5-27

\section{$\underline{\text { Investigación }}$}

Recibido:25-01-2018

\section{RESUMEN}

El problema abordado en esta investigación se centra en la caracterización del aprendizaje del álgebra, específicamente, está dirigido a establecer los errores cometidos por los estudiantes del $3^{\circ}$ semestre de la Facultad de Ciencias de la Educación de la mención Matemática de la Universidad de Carabobo en el aprendizaje de la teoría de conjunto. El marco teórico que se asume es la teoría de Errores en el aprendizaje de la Matemática, la metodología se basó en el paradigma cuantitativo sustentada en una investigación de campo tipo descriptivo; los datos se tomaron mediante la recolección de artefactos (Lanksshear y Knobel, 2000). Se proyecta que esta investigación contribuya con la sistematización de los errores en el aprendizaje de la teoría de conjunto de los estudiantes universitarios, y que sirva como punto de partida para generar una propuesta didáctica que proyecte la superación de los errores.

Palabras Clave: estudiantes de educación matemática, teoría de conjunto, errores

\section{Mariela Lilibeth Herrera" \\ Celsa Álvarez": \\ Universidad de Carabobo}

Aceptado:03-10-2018

\begin{abstract}
The problem addressed in this research focuses on the characterization of the learning of algebra, specifically, is directed to establish the errors committed by the students of the 3rd semester of the Faculty of Sciences of the education of the mention Mathematics of the University of Carabobo in learning the whole theory. The theoretical framework that is assumed is the theory of errors in the learning of mathematics, the methodology was based on the quantitative paradigm supported by a field investigation descriptive type; Data were collected by collecting artifacts (Lanksshear and Knobel, 2000). It is projected that this research contributes with the systematization of the errors in the learning of the theory of whole of the university students, and that it serves as starting point to generate a didactic proposal that projects the overcoming of the Errors.
\end{abstract}

Key words: Math education students, set theory, errors

\footnotetext{
* Profesora de Matemática, Magister en Educación Matemático. Especialista en Tecnología de la Computación en Educación, Estudiante de Doctorado en Educación Matemático. marielalilibeth@ gmail.com

** Licenciada en Educación mención Matemática, Magíster en Gerencia Avanzada en Educación, Coordinadora de la Mención Matemática de la Facultad de Educación de la Universidad de Carabobo. profecelsa@ hotmail.com
} 
CATÁLOGO DE ERRORES ASOCIADOS AL APRENDIZAJE DE LA TEORIA DE CONJUNTOS

Mariela Lilibeth Herrera y Celsa Álvarez

Pp 5-27

\section{A MODO DE INTRODUCCIÓN}

Partiendo de la idea, que el error forma parte del proceso de enseñanza y aprendizaje de cualquier disciplina; el cual, no sólo ha de ser considerado como la presencia de un esquema cognitivo inadecuado en el estudiante o como producto de una falta específica de conocimientos, puede ser visto como una herramienta que ayude al docente a organizar estrategias para reforzar los aspectos que generan mayor dificultad, y contribuyen a una corrección de los errores localizados.

En particular, la construcción del saber matemático requiere, por parte de los estudiantes, de reorganización e incremento de los conocimientos previos y, por parte de los educadores, la detección de las carencias, dificultades y errores que impiden que los conocimientos presentes en los estudiantes sean significativos. A este respecto Rico (1995) comenta:

Si los errores son elementos usuales en nuestro camino hacia el conocimiento verdadero, se puede concluir que en el proceso usual de construcción de los procesos matemáticos va a aparecer de formas sistemática los errores, y por lo tanto el proceso de construcción deberá incluir su diagnóstico, detención, corrección y superación mediante actividades que promuevan el ejercicio de la crítica sobre las propias producciones (p. 69).

Se hace necesario construir el conocimiento de cada noción matemática procurando facilitar la comprensión de los estudiantes, partiendo de que el propio constructo matemático es la esencia para decidir sobre la didáctica a utilizar en el aula, reflexionando acerca de cómo el conocimiento limita y condiciona el trabajo docente y el de los educadores matemáticos que se están formando, en particular en los tópicos de álgebra, porque justamente, la mayoría de objetos, procesos y conceptos manipulados en la educación secundaria de todos los niveles y modalidades están relacionados con la teoría de conjunto, como las soluciones de ecuaciones e inecuaciones; de aquí surge, entonces la necesidad considerar el tratamiento didáctico de los contenidos básicos del álgebra.

Desde la década de los 90 comenzó un creciente interés por el estudio del error, al respecto, Rico y Castro (1994) afirman que "El estudio de errores y dificultades de comprensión de los 
CATÁLOGO DE ERRORES ASOCIADOS AL APRENDIZAJE DE LA TEORIA DE CONJUNTOS

Mariela Lilibeth Herrera y Celsa Álvarez

Pp 5-27

escolares constituye una línea de investigación potente y productiva en Educación Matemática” (p.1). Además, Rico (1995) alude posteriormente que "El campo de estudio sobre errores en el aprendizaje de las matemáticas escolares hemos visto que se viene desarrollando y definiendo de manera crecientemente productiva durante los últimos años” (pp.24, 25).

En la actualidad, en la página web del "Grupo de Pensamiento Numérico y Algebraico" de la Universidad de Granada en España se consiguen once investigaciones referidas a los errores. Es importante destacar que el análisis de los errores involucra tanto el rol del docente, del estudiante, como los contenidos, los conocimientos previos requeridos, la institución, y el currículo, pues los errores están basados en conocimientos y experiencias previas que pueden tener diferentes factores que lo originan, entre lo que podemos citar: los obstáculos didácticos, cognitivos y epistemológicos, así como las deficiencias en los conocimientos previos asociadas a la noción en estudio. (Socas, 1997)

Los diversos modelos educativos que se han implementado en el marco de la modernización de la educación, buscan mejorar su calidad, potenciar el aprendizaje en todas las etapas escolares; disminuir el fracaso escolar y formar estructuras que permitan al estudiante desenvolverse posteriormente como adultos en la sociedad que les tocará vivir. Al respecto, la investigación de Herrera (2011) sugiere que:

Desde la perspectiva docente los errores se exteriorizan a través de resultados incorrectos, y la sola corrección lleva a explicitar la realización de las operaciones. Sin embargo, la posible vinculación entre ellos y las características de los educandos permitirá redimensionar tanto su tratamiento como su abordaje, que suponga la transformación de los modelos mentales de los estudiantes. (p.34)

El aporte básico de esta investigación, y así lo expresa su objetivo, es la recopilación de los errores producidos por los estudiantes durante el aprendizaje de la teoría de conjunto, conocimiento que puede ser empleado por los profesores desde una perspectiva didáctica para adelantarse a las posibles inconsistencias en las puedan incurrir los alumnos y poder tomar las previsiones necesarias, y de esta manera trazar estrategias que puedan socavar dicho error. 


\section{REFERENTES TEÓRICOS}

\section{Errores en el aprendizaje de la matemática}

La palabra error se deriva del latín ōris, tiene distintas aserciones en el Diccionario esencial de la Lengua Española (2006), se le vincula al problema de la verdad, con la fuente del conocimiento, con desviación o uso incorrecto de una norma, acción desacertada o equivocada, conocimiento falso o disparidad entre el conocimiento y la realidad de las cosas, concepto errado; mientras que en el campo de la matemática y física se relaciona con el proceso de medición y se concibe como la diferencia entre el valor obtenido a partir de una medición o un cálculo y el valor real.

Ferrater Mora (2004) realiza una revisión filosófica del error, donde se resaltan los aspectos más relevantes para este trabajo: Aristóteles al estudiar la presencia del error en el juicio explica que puede estar relacionado con la visión de los términos o con el reflexión enunciada sobre ellos, pudiéndose presentar la conjunción de ambos, alcanzando el primero un ámbito general y el segundo sólo particular. Por su parte, Sócrates propone la autocrítica y la crítica racional como mecanismos para examinar los errores procurando una verdad objetiva. Para los escolásticos el error, es discordancia, divergencia, inverso a la verdad, es revelado desde la óptica de la certidumbre, para entender el error debemos ser conscientes de las variadas formas en que se puede presentar la verdad, y su origen concierne a la estructura lógica, psicológica, o simple ignorancia. Para los filósofos modernos sólo se puede llegar a la verdad previa eliminación del error; mientras Descarte explica el error desde la voluntad, plantea que se encuentra en el acontecimiento de la voluntad que se emite sobre el juicio y no en el juicio, y al igual que Leibniz lo contempla como ausencia de inteligencia, otra enfoque considera el error como el enlace de la voluntad y del mundo de la idea.

Popper citado por Rico (1995) contempla la presencia del error a pesar de la verdad visible, y expone que la verdad puede hallarse y desaparecer con facilidad, imputa a los errores un gran 


\section{CATÁLOGO DE ERRORES ASOCIADOS AL APRENDIZAJE DE LA TEORIA DE CONJUNTOS}

Mariela Lilibeth Herrera y Celsa Álvarez

Pp 5-27

poder de conservación, es decir, se requiere una revisión constante de las teorías para identificar la verdad mediante la descubrimiento y eliminación del error, expone que el conocimiento no surge de la nada, sino que es una gran construcción que se progresa a través de la modificación del anterior, valiéndose de algunos criterios para revelar el error.

Socas (1997) explica que para Lakatos, los errores encontrados en una teoría permiten su trasformación o ampliación, errores cometidos en el desarrollo histórico de un conocimiento son considerado como concepciones limitadas, específicamente, expone:

Algunos autores como Lakatos, prefieren considerar otros errores como "concepciones limitadas", matiz totalmente válido, pues decimos que algún procedimiento es correcto o no, a partir de los elementos que conforman las teorías actuales, pero con ello cometemos el error de hacer juicios con marcos de referencia que no corresponden a la situación que se analiza".

Esta matiz de "concepción limitada" que se le da a los errores en la historia de las matemáticas, puede ser válido también en el caso de los errores cometidos por los estudiantes, puesto que muchos de éstos pueden explicarse a través de los métodos que ellos desarrollan con el tiempo, siendo dichos métodos válidos en algunos casos solamente.(p.139)

Hemos visto como el error está presente en el desarrollo científico desde tiempos ancestrales, es más, el error, como toda actividad humana, está vinculado al proceso de enseñanza-aprendizaje, aunque es concebido negativamente. Los errores son asociados a un aprendizaje deficiente de los conocimientos previos, a un escaso manejo de destrezas elementales, a un esquema cognitivo inadecuado y no solamente como consecuencia de una falta de conocimiento, sino, que además, los alumnos ocultan serios errores conceptuales que dificultaran el aprendizaje subsiguiente.

En la enseñanza, habitualmente, el docente no saca provecho del error, sino que pretende que no aflore, y si lo hace, lo erradica inmediatamente, ya que para éste el error está relacionado con la falta de comprensión, porque el error en la enseñanza es la diferencia entre lo que el profesor desea como respuesta del alumno y la que éste le suministra. Pessoa (1997) ha 


\section{CATÁLOGO DE ERRORES ASOCIADOS AL APRENDIZAJE DE LA TEORIA DE CONJUNTOS}

Mariela Lilibeth Herrera y Celsa Álvarez

Pp 5-27

observado que para el profesor es difícil trabajar con el error de sus alumnos para transformarlo en situación de aprendizaje donde debe dejar claro lo que es correcto y lo que está errado.

Los errores están interiorizados en los alumnos y no son fáciles de eliminar, resurgen al poco tiempo, el estudiante debe participar activamente en el proceso de superar sus propios errores, el profesor debe provocar conflictos en su mente a partir de la inconsistencia de sus propios errores colocándoles en situaciones de reflexión sobre sus ideas erróneas, para que

intente comprender y darle significado a los objetos matemáticos, forzándolo a participar activamente en la solución del conflicto. En este sentido la noción de error está relacionada con la noción de obstáculo epistemológico desarrollada por Bachelard (2007):

... se conoce afrontando un conocimiento anterior, destruyendo los conocimientos mal adquiridos o superando aquello que en el espíritu mismo obstaculiza la espiritualización. Un obstáculo epistemológico se incrusta en el conocimiento no formulado. Costumbres intelectuales que fueron útiles y sanas, pueden después de un tiempo obstaculizar la investigación. (pp. 15-16)

Los errores pueden relacionarse con diversas causas, incomprensión de la teoría, dificultad con los cálculos, aversión hacia el contenido o la propia disciplina, un algoritmo mal aplicado, se pueden percibir como la expresión exterior de un complejo proceso en el que se enlazan: el docente, el estudiante, el contexto, la complejidad propia del contenido matemático, el currículo, el contexto educativo, e incluso, los factores afectivos y motivacionales del individuo, así como sus habilidades lógicas-matemáticas.

\section{Características fundamentales de los errores}

Mulhern (citado en Rico, 1995) caracteriza los errores en cuanto al surgimiento como espontáneos e inesperados por el profesor, son de carácter persistentes e individuales y difíciles de eliminar porque requieren de una reorganización de los conocimientos en el alumno, y detalla las siguientes como características: 


\section{CATÁLOGO DE ERRORES ASOCIADOS AL APRENDIZAJE DE LA TEORIA DE CONJUNTOS}

Mariela Lilibeth Herrera y Celsa Álvarez

Pp 5-27

1. Los errores son sorprendentes. Con frecuencia los errores cometidos por los alumnos surgen de manera sorprendente, ya que por lo general se han mantenido ocultos para el profesor durante algún tiempo.

2. Los errores son a menudo extremadamente persistentes, debido a que pueden reflejar el conocimiento de los alumnos sobre un concepto o un uso particular de reglas nemotécnicas. Son resistentes al cambiar pr sí mismos ya que la corrección de errores puede necesitar de una reorganización fundamental del conocimiento de los alumnos.

3. Los errores pueden ser o bien sistemáticos o por azar. Los primeros son muchos más frecuentes y, por lo general, más efectivos para revelar los procesos mentales subyacentes; estos errores se toman como síntomas que señalan hacia un método o comprensión equivocada subyacente, que el estudiante considera y utiliza como correcto. Los errores por azar reflejan falta de cuidado y lapsus ocasionales, y tienen relativamente poca importancia

4. Los errores ignoran el significado; de modo, respuestas que son obviamente incorrectas, no se ponen en cuestión. Los alumnos que cometen un error no consideran el significado de los símbolos y conceptos con los que trabajan. (p.9)

En virtud, de que en este artículo, sólo expondremos la lista de los errores encontrados durante el aprendizaje de la teoría de conjuntos, sólo consideraremos como referentes teóricos las aserciones dadas al error por filósofos y matemáticos, y las características expuestas por Mulher; es importante destacar que no categorizaremos los errores encontrados, por lo cual no hacemos mención a las taxologías existentes.

\section{REFERENTES METODOLÓGICOS}

Este trabajo se realizó bajo el paradigma cuantitativo, y corresponde a una investigación de campo descriptiva, la cual según Balestrini (2002) consiste en:

La descripción acerca de singularidades de una realidad estudiada, podrá estar referida a una comunidad, una organización, un hecho delictivo, las características de un tipo de gestión, conducta de un individuo o grupales, comunidad, de un grupo religioso, electoral, etc. Por lo tanto, su esquema de investigación en cuanto a su contenido, será diferente a los estudios de comprobación de hipótesis causales, mucho más riguroso, que permiten inferir 


\section{CATÁLOGO DE ERRORES ASOCIADOS AL APRENDIZAJE DE LA TEORIA DE CONJUNTOS}

Mariela Lilibeth Herrera y Celsa Álvarez

Pp 5-27

acerca de la casualidad, y en los cuales se aumenta el grado de fiabilidad y se reducen las inclinaciones. (p.6)

La muestra de la investigación la conformaron los cuarenta y tres (43) educandos del curso de Álgebra I de la Facultad de Educación de la Universidad de Carabobo, durante el semestre 2016-1. Se utilizó la recolección de artefactos, en este caso las pruebas escritas realizadas para la evaluación de la asignatura, como la técnica para la recoger los datos.

Mulhern (citado en Rico, 1997) plantea cuatro categorías que han surgido, desde las distintas investigaciones realizadas en el ámbito de la educación matemática, como técnica de análisis para el estudio de los errores, estando esta investigación enmarcada, en la primera que consiste en "Contar simplemente el número de soluciones incorrectas a una variedad de problemas. Este método, que tiene un valor diagnostico limitado es cercano al método psicométrico" (p.22), además, se hace uso de la estadística descriptiva para presentar los datos en cuadros y establecer la frecuencia porcentual de las respuestas dadas por los estudiantes a las diversas preguntas de las evaluaciones, para finalmente, inferir lo que realiza el estudiante a través de la descripción de lo que observan las investigadoras que realizan los individuos en estudio.

\section{PRESENTACIÓN Y ANÁLISIS DE LOS RESULTADOS}

A continuación se muestran los datos recabados a través de la primera evaluación de la siguiente manera: se describe cada instrumento con el contenido a evaluar, mientras que, la información obtenida de cada una de las interrogantes de la evaluación se separa en dos partes: (1) la síntesis de las respuestas escritas por los estudiantes (Primera Información), y (2) las consideraciones realizadas por las investigadoras (Inferencias de las Investigadoras), lo concerniente a las otras dos evaluaciones se omiten en este artículo por cuestión de espacio, sin embargo, se consideró importante listar los errores encontrados en las evaluaciones omitidas. 


\section{CATÁLOGO DE ERRORES ASOCIADOS AL APRENDIZAJE DE LA TEORIA DE CONJUNTOS}

Mariela Lilibeth Herrera y Celsa Álvarez

Pp 5-27

Instrumento No.1: PRUEBA ESCRITA No.1

Descripción del instrumento: se trató de una prueba escrita de 9 preguntas, totalmente práctica, no incluía definiciones ni completación. Contenido a evaluar: relación de pertenencia e inclusión, determinación de conjunto por extensión y por comprensión, diagramas de Venn, complemento de un conjunto y conjunto de partes.

(1) Primera Información: A continuación (en la tabla No.1) se muestra la cantidad de respuestas dadas por los estudiantes a la pregunta:

Considere los siguientes conjuntos $A=\{1,2,3,4\} ; B=\{\{1\},\{1,2\}, 3\} ; C=\{1,2\} ; D=\{1,2,3,4,5\} ; E=\{\{1\}\}$. Completa la siguiente tabla utilizando los símbolos $\subset, \not \subset, \in$ ó $\notin$ que corresponda para expresar la relación establecida: (0.1c/u total 2 puntos)

\begin{tabular}{|c|c|c|c|c|c|}
\hline & A & B & C & D & E \\
\hline 1 & & & & & \\
\hline 2 & & & & & \\
\hline$\{1\}$ & & & & & \\
\hline$\{1,2\}$ & & & & & \\
\hline
\end{tabular}

Tabla 1

Cantidad de respuestas referidas a la relación de pertenencia e inclusión

\begin{tabular}{|c|c|c|c|c|c|c|c|c|c|c|c|c|c|c|c|c|c|c|c|c|}
\hline & \multicolumn{4}{|c|}{ A } & \multicolumn{4}{|c|}{ B } & \multicolumn{4}{|c|}{ C } & \multicolumn{4}{|c|}{ D } & \multicolumn{4}{|c|}{$\mathbf{E}$} \\
\hline & $\in$ & $\notin$ & $\subset$ & $\not \subset$ & $\in$ & $\notin$ & $\subset$ & $\not \subset$ & $\in$ & $\notin$ & c & $\not \subset$ & $\in$ & $\notin$ & $c$ & $\not \subset$ & $\in$ & $\notin$ & $\subset$ & $\not \subset$ \\
\hline 1 & 12 & 0 & 5 & 0 & 1 & 11 & 3 & 2 & 14 & 1 & 2 & 0 & 13 & 0 & 4 & 0 & 0 & 13 & 1 & 3 \\
\hline 2 & 12 & 0 & 5 & 0 & 1 & 11 & 0 & 5 & 13 & 1 & 3 & 0 & 13 & 0 & 4 & 0 & 0 & 14 & 0 & 3 \\
\hline$\{1\}$ & 0 & 6 & 2 & 9 & 5 & 0 & 12 & 0 & 0 & 5 & 2 & 10 & 0 & 5 & 3 & 9 & 9 & 0 & 8 & 0 \\
\hline$\{1,2\}$ & 0 & 5 & 3 & 9 & 8 & 0 & 9 & 0 & 5 & 1 & 6 & 5 & 0 & 3 & 4 & 10 & 1 & 4 & 0 & 12 \\
\hline
\end{tabular}

Inferencias de las Investigadoras: Como en el tabla se proponían cuatro opciones $(\in, \notin$, $\subset, \not \subset)$ de respuestas: dos referidas a la pertenencia y dos a la inclusión, hay dos opciones correctas y dos incorrectas. El 55\% de las respuestas dadas se corresponden a la relación de pertenencia, y el 95\% fueron correctas, siendo el error más frecuente que un conjunto pertenece a sí mismo. Sin embargo, de las respuestas dadas en función de la relación de inclusión, sólo el $29 \%$ fueron correctas, mostrando los estudiantes la mayor dificultad en la inclusión de conjuntos. Entre los errores encontramos: $\mathrm{A} \in \mathrm{A}$ (en lugar de que $\mathrm{A} \subset \mathrm{A}$ ); cuando los elementos de un 


\section{CATÁLOGO DE ERRORES ASOCIADOS AL APRENDIZAJE DE LA TEORIA DE CONJUNTOS}

Mariela Lilibeth Herrera y Celsa Álvarez

Pp 5-27

conjunto son a su vez conjuntos emplean la relación de inclusión para denotar pertenencia; no reconocen los subconjuntos propios de un conjunto; consideran los elementos como subconjuntos; no reconocen la propiedad, de que todo conjunto es subconjunto de sí mismo.

Alumno 1

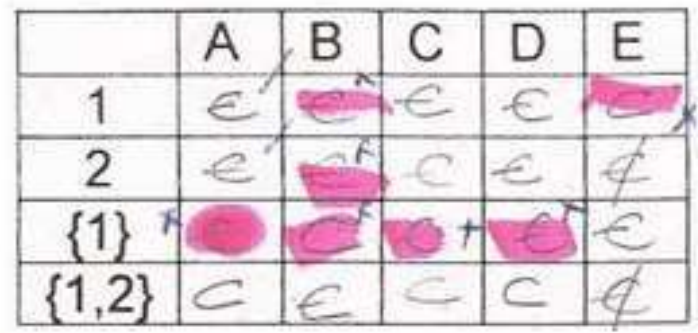

Alumno 3

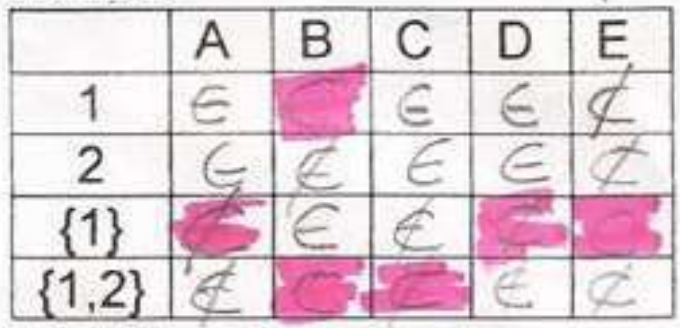

Alumno 2

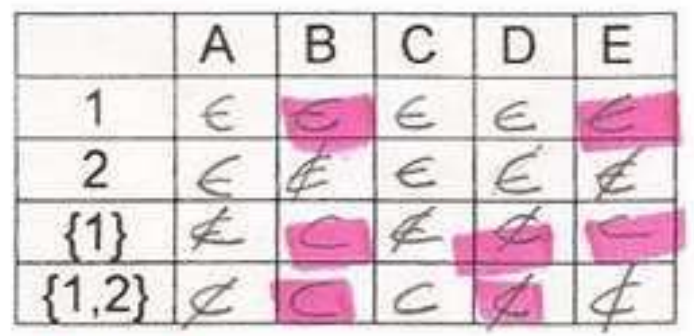

Alumno 4

\begin{tabular}{|c|c|c|c|c|c|}
\hline & $A$ & $B$ & $C$ & $D$ & $E$ \\
\hline 1 & $\epsilon$ & $\notin$ & $\epsilon$ & $E$ & $\epsilon$ \\
\hline 2 & $\epsilon$ & $\epsilon$ & $\epsilon$ & $E$ & $\epsilon$ \\
\hline$\{1\}$ & $E$ & $\epsilon$ & $E$ & $E$ & $E$ \\
\hline$\{1,2\}$ & $\epsilon$ & $\epsilon$ & $C$ & $£$ & $\notin$ \\
\hline
\end{tabular}

Figura 1. Ejemplos de las respuesta descritas en la Tabla 1

(2) Primera Información: en la siguiente pregunta, cuatro (4) conjuntos estan determinados por extensión y los alumnos deben bosquejarlos en diagramas de Venn:

Represente los conjuntos A, B, C, D y su relación en un diagrama de Venn, siendo $A=\{1,2,3,4\}$; $\mathrm{B}=\{\{7\}, 1,2,3\} ; \mathrm{C}=\{1,2,8,9\} ; \mathrm{D}=\{1,2,3,4,5,6\}$ : (2 puntos)

En relación a los errores de esta pregunta, encontramos que: los estudiantes escriben el signo igual después del conjunto al hacer el diagrama de Venn, escriben los conjuntos con letras minúsculas, no consideran todos los elementos de los conjuntos, repiten los elementos en la representación, intercambian el nombre de los conjuntos, no dibujan óvalos cerrados, no encierran al número 7 en un óvalo dentro de B, pues no saben como representar los elementos que a su vez son conjuntos, no identifican el conjunto (olvidan colocarle el nombre). 


\section{CATÁLOGO DE ERRORES ASOCIADOS AL APRENDIZAJE DE LA TEORIA DE CONJUNTOS}

Mariela Lilibeth Herrera y Celsa Álvarez

Pp 5-27

(3) Primera Información: El docente escribió la propiedad transitiva de la inclusión de conjuntos y los alumnos debían construir un contraejemplo para la implicación recíproca asociada.

Dar contraejemplo para el recíproco del teorema: $(\mathrm{A} \subset \mathrm{B} \wedge \mathrm{B} \subset \mathrm{C}) \Rightarrow \mathrm{A} \subset \mathrm{C}$ (2 puntos)

Recíproco:

Contraejemplo:

En las respuestas escritas por los estudiantes, notamos que, no escriben el recíproco en forma correcta, sólo escriben la tesis y no colocan la implicación, escriben el recíproco correctamente pero el contraejemplo lo escriben del directo (a pesar de que el directo es una propiedad), como contraejemplo escriben la negación del recíproco, sólo consideran una condición en la tesis del recíproco, niegan la conjunción. Inferencias de las Investigadoras: los estudiantes no formulan la implicación recíproca, puede ser porque no han comprendido cuál es el antecedente y el consecuente de una implicación, o, como el antecedente involucraba una conjunción los estudiantes no identificaron la implicación como el conectivo principal.

(4) Primera Información: El docente escribió por extensión el conjunto formado por un número irracional y su opuesto, los estudiantes debían escribir el conjunto por comprensión. Expresar por comprensión el conjunto: $\mathrm{D}=\{-\sqrt{3}, \sqrt{3}\}$

En cuanto a la simbología, se observa que unos estudiantes no emplean el signo igual ni las llaves para denotar los conjuntos, otros no escriben las llaves de cierre, al definir el conjunto por comprensión no identifican al conjunto universal, otros alumnos no separan el conjunto universal de la condición con el "tal que" $(/,:)$, algunos escriben el conjunto de los naturales o de los enteros como conjunto referencial, denotan al elemento genérico con una letra y para enunciar la propiedad común a todos los elementos del conjunto emplean otra.

Inferencias de las Investigadoras: los estudiantes identifican los números irracionales como subconjunto de los naturales y de los enteros, el mal uso de los signos de puntuación en la escritura conlleva a los estudiantes a generalizar su mal uso en el lenguaje matemático, 


\section{CATÁLOGO DE ERRORES ASOCIADOS AL APRENDIZAJE DE LA TEORIA DE CONJUNTOS}

Mariela Lilibeth Herrera y Celsa Álvarez

Pp 5-27

relacionan el signo igual sólo con las operaciones matemáticas, por lo que no consideran necesario escribirlo para denotar un conjunto por comprensión.

(5) Primera Información: en esta pregunta se escribió un conjunto por comprensión y los estudiantes debían escribirlo por extensión. Expresar por extensión el conjunto $\mathrm{H}=\{\mathrm{x} \in \mathrm{N} /(\mathrm{x}+3)(\mathrm{x}+2)=0\}$

Como respuesta, los estudiantes escribieron números naturales, otros, escribieron números enteros (negativos), otros escribieron al conjunto vacío entre llaves, es decir, escribieron como solución un conjunto unitario cuyo único elemento era el conjunto vacío, otros, escribieron como respuesta un conjunto unitario cuyo único elemento era el cero. Hay alumnos que para denotar un conjunto por extensión escriben el conjunto referencial y luego escriben la variable seguido del signo igual y mencionan los elementos. Inferencias de las Investigadoras: los estudiantes no tienen claro la anidación de los conjuntos numéricos, no perciben el hecho de que un conjunto escrito por comprensión tiene dos condiciones: un conjunto referencial y un criterio de pertenencia para estar en el conjunto, además, dichas condiciones se deben cumplir simultáneamente. Los estudiantes mezclan las características de un conjunto escrito por comprensión y por extensión, además cambian el conjunto referencial por otro donde se encuentran los elementos que ellos quieren (no son correctos) listar.

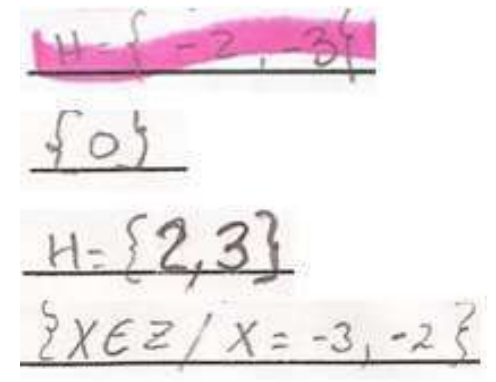

Figura 2. Ejemplo de los conjuntos escritos por los estudiantes

(6) Primera Información: En esta pregunta los alumnos tenían que determinar el complemento del conjunto (escrito por comprensión) respecto a los números reales, empleando como criterio 


\section{CATÁLOGO DE ERRORES ASOCIADOS AL APRENDIZAJE DE LA TEORIA DE CONJUNTOS}

Mariela Lilibeth Herrera y Celsa Álvarez

Pp 5-27

de pertenencia las soluciones reales de una inecuación dada con valor absoluto. Determina el complemento del conjunto respecto a los números reales, si $\mathrm{A}=\left\{\mathrm{x} \in \mathrm{R} /\left|x-\frac{1}{2}\right| \leq 2\right\}:(2$ puntos $)$

Tienen dificultad para establecer gráficamente que $\frac{-3}{2} \leq x$, no lo relacionan con que $x \geq \frac{-3}{2}$, colocan el universo donde debe ir el conjunto y el conjunto en lugar del universo (escriben $\mathrm{C}_{\mathrm{A}} \mathrm{R}$ en lugar de $\mathrm{C}_{\mathrm{R}} \mathrm{A}$ ), consideran sólo parte de la solución, pues la solución es la unión de dos conjuntos, colocan corchete para expresar el intervalo solución pero el conjunto es abierto, grafican correctamente pero no establecen el intervalo solución, confunden el extremo inferior con el superior, escriben $\mathrm{a} \leq \mathrm{x} \geq \mathrm{b}$, se les olvida la variable $\mathrm{x} \geq \mathrm{a} \wedge \leq \mathrm{b}$. Inferencias de las Investigadoras: en la solución de la pregunta, el alumno se enfrenta con temas que serán explicados con detalle posteriormente como la unión de dos conjuntos y los intervalos abiertos y cerrados, la dificultad demostrada por los estudiantes en la mayoría se relaciona con las inecuaciones en $\mathrm{R}$, más que en el complemento de un conjunto.

(7) Primera Información: se presentaron 4 conjuntos en diagramas de Venn para que los estudiantes determinaran esos conjuntos por extensión y por comprensión. Al escribir los conjuntos por extensión no colocan el signo igual, además escriben un conjunto referencial y luego escriben la variable seguido de dos puntos y comienzan a listar los elementos del conjunto. Partiendo del siguiente diagrama de Venn:

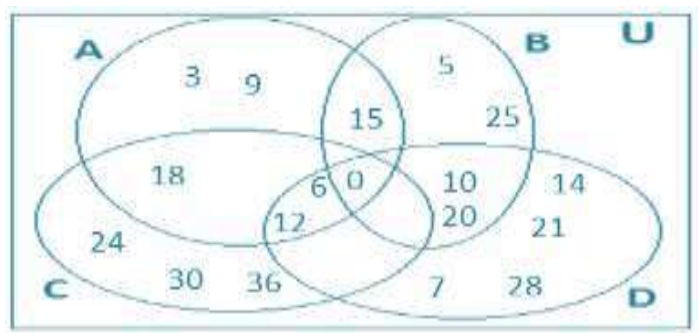

Determina por comprensión los conjuntos A y B: (1c/u total 2 puntos) Determina por extensión los conjuntos C y D: (1c/u total 2 puntos) 


\section{CATÁLOGO DE ERRORES ASOCIADOS AL APRENDIZAJE DE LA TEORIA DE CONJUNTOS}

Mariela Lilibeth Herrera y Celsa Álvarez

Pp 5-27

En cuanto a los errores encontrados, podemos citar que los alumnos al escribirlos los conjuntos por extensión colocan dos elementos genéricos distintos: uno para denotar la relación con el conjunto universal y otro elemento (distinto al anterior) para denotar la condición que deben cumplir los elementos para pertenecer al conjunto; algunos estudiantes no saben cómo expresar en lenguaje matemático a los múltiplos de un determinado número, para escribir los múltiplos de tres escriben los números impares, otros emplean ambos signos de desigualdad en una misma expresión matemática $(0 \leq n \geq 6)$, existen otros alumnos que emplean a $\mathrm{x}$ como elemento genérico y a $\mathrm{P}(\mathrm{x})$ para expresar la condición que deben cumplir los elementos del conjunto.

Inferencias de las Investigadoras: mezclan la forma de escribir un conjunto por extensión y comprensión, puede ser, debido a que hay conjuntos que aceptan ser escritos tanto por extensión como por comprensión; el hecho de que en bachillerato ya no se practique cómo pasar del lenguaje escrito a la simbología matemática influye en que los estudiantes no sepan escribir los múltiplos de un determinado número, confunden elemento genérico con variable, por lo que escriben todas las letras que intervienen en el criterio de pertenencia del conjunto como "elementos genéricos" del conjunto, emplean la simbología de la función proposicional como variable proposicional se debe a la explicación del manual de Álgebra I que es considerado el libro de texto.

Zile y Nuñez (2008) definen que un conjunto está determinado por comprensión:

Cuando se da un criterio de pertenencia que permita decidir si un elemento pertenece o no al conjunto considerado. En este caso se representa cerrado entre llaves y dentro de ellas anotaremos con una letra minúscula a un elemento que representa a todos los elementos del conjunto, que llamaremos elemento genérico, luego una barra así: / que se le "tal que" o "tales que", y a continuación el criterio de pertenencia, o la propiedad que cumple el representante de todos los elementos del conjunto, así: $\mathrm{M}=\{\mathrm{x} / \mathrm{P}(\mathrm{x})\}$. (p.6) 


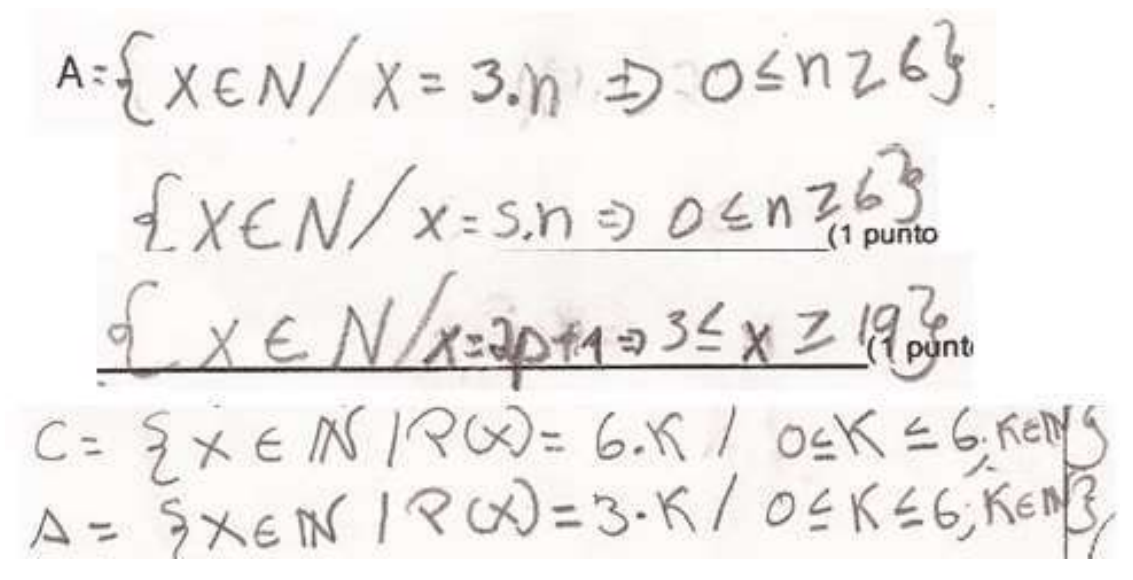

Figura 3. Ejemplo de los conjuntos escritos por los estudiantes a partir de los diagramas de Venn

\section{(8) Primera Información:}

Considere el conjunto $\mathrm{B}=\{\mathrm{f},\{\mathrm{f}\}\}$, hallar $P(\mathrm{~B})$ y $P(P(\mathrm{~B}))$ : (4 puntos)

Los alumnos no consideran a $\phi$ como un elemento de $\mathrm{P}(\mathrm{B})$, consideran a $\mathrm{f} \in \mathrm{B}$ como un elemento de $\mathrm{P}(\mathrm{B})$, escriben $\{\phi\} \subset \mathrm{P}(\mathrm{P}(\mathrm{B}))$ cuando deben decir que $\{\phi\} \in \mathrm{P}(\mathrm{P}(\mathrm{B}))$, escriben $\mathrm{P}(\mathrm{P}(\mathrm{B}))=$ cada vez que van a nombrar un elemento como si se estuviesen hablando de un conjunto unitario, en $\mathrm{P}(\mathrm{P}(\mathrm{B}))$ tienden a colocarle más llave a cada elemento de lo debido, al listar los elementos de $\mathrm{P}(\mathrm{B})$ establecen una especie de transitividad empleando los signos $\subset \mathrm{y} \in,\{\mathrm{f}\} \subset \mathrm{B} \in \mathrm{P}(\mathrm{B})$, a pesar de que escriben bien a $\mathrm{P}(\mathrm{B})$ quieren incluir los elementos de $\mathrm{P}(\mathrm{B})$ en $\mathrm{P}(\mathrm{P}(\mathrm{B}))$, relacionan los elementos de $\mathrm{P}(\mathrm{B})$ y de $\mathrm{P}(\mathrm{P}(\mathrm{B})$ ) aumentando el número de llaves, igualan el conjunto a su cardinal, para decir que $\mathrm{P}(\mathrm{B})$ tiene cuatro elementos escriben $\mathrm{P}(\mathrm{B})=4$.

Inferencias de las Investigadoras: el conjunto de partes es un tema nuevo para los estudiantes, para comprender el conjunto de partes hay que tener claro la relación de pertenencia y de inclusión, sin embargo, los estudiantes han demostrado dificultades con estas dos relaciones; debido a que el conjunto dado tenía dos elementos, cuando relacionan los elementos de $\mathrm{P}(\mathrm{B})$ y de $\mathrm{P}(\mathrm{P}(\mathrm{B})$ ) aumentando el número de llaves están desconociendo a los subconjuntos impropios de un conjunto, además, están generalizando este caso al considerar como únicos subconjunto de $\mathrm{P}(\mathrm{B})$ a los elementos de $\mathrm{P}(\mathrm{B})$ encerrados entre llaves. 


\section{CATÁLOGO DE ERRORES ASOCIADOS AL APRENDIZAJE DE LA TEORIA DE CONJUNTOS}

Mariela Lilibeth Herrera y Celsa Álvarez

Pp 5-27

\section{(9) Primera Información:}

Demostrar la veracidad o falsedad de la siguiente proposición: $(A \neq B \wedge B \not \subset C) \Rightarrow A \neq C$ (2 puntos) Los estudiantes escribieron que la afirmación era cierta y construyeron un contraejemplo para demostrarlo, para construir un contraejemplo consideraban a $\mathrm{B}=\phi$ y esto conlleva a que $\mathrm{B} \subset \mathrm{C}$, realizaron diagramas de Venn para construir un contraejemplo pero consideraron a A y B incluidos en C. Inferencias de las Investigadoras: al intentar demostrar una afirmación en función de un sólo caso están ejemplificando por lo que subyace la idea en el alumno que si se cumple para uno se cumple para todos, al afirmar la proposición e intentar construir un contraejemplo demuestran incomprensión de los métodos de demostración, posiblemente porque esta actividad desapareció de los contextos de las clases de Matemática de la educación media general, si bien pueden emplear diagramas de Venn para elaborar un contraejemplo se deben cumplir todas las hipótesis dadas en la proposición, sin embargo, sólo consideran la primera proposición de la conjunción dada como hipótesis.

A continuación se listan, de forma resumida los distintos errores encontrados en las tres evaluaciones realizadas con respecto al tema de teoría de conjuntos en la asignatura Álgebra I en la mención de Matemática de la Facultad de Educación de la Universidad de Carabobo:

(1) Uso de la relación de inclusión $(\subset)$ para denotar pertenencia $(\in)$

(2) Uso de la relación de pertenencia $(\in)$ para denotar inclusión $(\subset)$

(3) No identifican los subconjuntos propios de un conjunto

(4) Consideran los elementos como subconjuntos

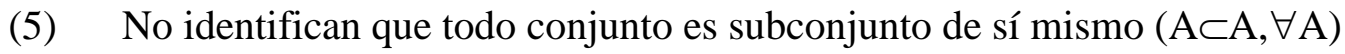

(6) No dibujan figuras cerrados para representar diagramas de Venn

(7) No dibujan los elementos que a su vez son conjuntos en un óvalo al emplear diagramas de Venn

(8) No escriben todos los elementos de los conjuntos, al emplear diagramas de Venn

(9) No identifican el conjunto con la letra con que están denostados

(10) Ejecuta incorrectamente el cálculo de las raíces de un polinomio

(11) Identifican los números irracionales como subconjunto de los naturales

(12) Identifican los números irracionales como subconjunto de los enteros 
(13) Tienen dificultad para establecer gráficamente que $\frac{-\mathbf{3}}{\mathbf{2}} \leq x$

(14) Intercambian los nombres de los conjuntos

(15) Resuelven la desigualdad como si fuera una igualdad numérica

(16) No consideran a $\phi$ como un elemento de $\mathrm{P}(\mathrm{B})$

(17) En una desigualdad estricta consideran los extremos como parte de la solución

(18) No saben cómo expresar en lenguaje matemático a los múltiplos de un determinado número

(19) Grafican correctamente la solución de la inecuación pero no establecen el intervalo

(20) En un intervalo confunden el extremo inferior con el superior

(21) No emplean el cuantificador universal

(22) Consideran a los elementos $\mathrm{B}$ como un elemento de $\mathrm{P}(\mathrm{B})$

(23) Escriben $\{\phi\} \subset \mathrm{P}(\mathrm{P}(\mathrm{B}))$ en lugar de $\{\phi\} \in \mathrm{P}(\mathrm{P}(\mathrm{B}))$

(24) Formulan la transitividad de la relación de pertenencia $(A \in B \wedge B \in C \Rightarrow A \in C)$

(25) No escriben la palabra demostración

(26) Omiten justificaciones

(27) Realizan un ejemplo para demostrar la veracidad de la afirmación

(28) Realizan un contraejemplo errado para demostrar que la proposición es falsa.

(29) Realizan un diagrama de Venn para demostrar la veracidad de la proposición

(30) Escriben números negativos a ambos lados del origen del sistema de coordenadas en el eje de las ordenadas

(31) Emplean como elemento genérico a " $x$ " aunque el conjunto universal es $\mathrm{R}^{2}$

(32) Demuestran sólo una inclusión para demostrar la igualdad de dos conjuntos

(33) Emplean diagramas de Venn para ejemplificar la hipótesis pero escriben dos conjuntos B diferentes entre sí

(34) La afirmación no corresponde con la justificación

(35) No realizan conclusión al final de la demostración

(36) Graficar un intervalo abierto igual a uno cerrado

(37) Emplean llaves para denotar intervalos de números reales

(38) Emplean corchetes para escribir un conjunto unitario

(39) Incluyen los elementos de $\mathrm{P}(\mathrm{B})$ en $\mathrm{P}(\mathrm{P}(\mathrm{B}))$

(40) Escriben que la afirmación era cierta y construyeron un contraejemplo para demostrarlo

(41) Escriben $(\mathrm{x} \in \mathrm{A} \wedge \mathrm{x} \in \mathrm{B} \wedge \mathrm{x} \in \mathrm{C})$ por hipótesis y argumentan que por transitividad de la conjunción $\mathrm{A} \subset \mathrm{C}$

(42) Obvian condiciones de la hipótesis

(43) Grafican una recta en lugar de una circunferencia

(44) Escriben la ecuación de la recta en lugar de la circunferencia

(45) Escriben sólo asociatividad o conmutatividad como justificación

(46) Escriben R como conjunto referencial en lugar de $\mathrm{R}^{2}$

(47) No cierran completamente el signo del infinito $\propto$ 
CATÁLOGO DE ERRORES ASOCIADOS AL APRENDIZAJE DE LA TEORIA DE CONJUNTOS

Mariela Lilibeth Herrera y Celsa Álvarez

Pp 5-27

(48) Transforman defectuosamente la simbología algebraica, usan una coma en lugar del signo $\cup$

(49) Transforman defectuosamente la simbología, emplean ambos signos de desigualdad en una misma expresión matemática $(0 \leq n \geq 6)$

(50) Transforman defectuosamente la simbología algebraica, escriben $\mathrm{P}(\mathrm{P}(\mathrm{B}))=$ cada vez que van a nombrar un elemento como si se estuviesen hablando de un conjunto unitario

(51) Transforman defectuosamente la simbología algebraica, relacionan los elementos de $\mathrm{P}(\mathrm{B}) \mathrm{y}$ de $\mathrm{P}(\mathrm{P}(\mathrm{B}))$ aumentando el número de llaves

(52) Transforman defectuosamente la simbología algebraica, en $\mathrm{P}(\mathrm{P}(\mathrm{B}))$ tienden a colocarle más llave a cada elemento de lo debido

(53) Transforman defectuosamente la simbología algebraica, al listar los elementos de $\mathrm{P}(\mathrm{B})$ establecen una especie de transitividad empleando los signos $\subset \mathrm{y} \in,\{\mathrm{f}\} \subset \mathrm{B} \in \mathrm{P}(\mathrm{B})$

(54) Transforman defectuosamente la simbología, escriben el infinito abierto $(\propto)$

(55) Transforman defectuosamente la simbología, colocan corchete para expresar en intervalo abierto

(56) Transforman defectuosamente la simbología algebraica, para denotar pertenencia $(\in)$ emplean la letra e, E, E

(57) Transforman defectuosamente la simbología algebraica, para denotar inclusión $(\subset)$ emplean la letra $\mathrm{C}$

(58) Transforman defectuosamente la simbología algebraica, para negar la pertenencia o la inclusión emplean la tachadura de forma contrapuesta $(\in, \digamma)$

(59) Transforman defectuosamente la simbología algebraica, aal emplear diagramas de Venn, escriben el signo igual después de la letra que denota un conjunto

(60) Transforman defectuosamente la simbología algebraica, no emplean el signo igual ni las llaves para denotar los conjuntos

(61) Transforman defectuosamente la simbología algebraica, no escriben las llaves de cierre

(62) Transforman defectuosamente la simbología algebraica, no separan el conjunto universal de la condición con el "tal que" $(/,:$ )

(63) Transforman defectuosamente la simbología algebraica, denotan al elemento genérico con una letra y para enunciar la propiedad común a todos los elementos del conjunto emplean otra

(64) Transforman defectuosamente la simbología algebraica, partiendo de que $x \in A$ llegan a que $\mathrm{x} \in \mathrm{A}$, en lugar de " $\therefore$ " escriben “..."

(65) Uso inadecuado de la notación, al escribir conjuntos por extensión escriben un conjunto referencial y luego escriben la variable seguido de dos puntos y comienzan a listar los elementos del conjunto

(66) Uso inadecuado de la notación, escriben $\mathrm{C}_{\mathrm{A}} \mathrm{R}$ en lugar de $\mathrm{C}_{\mathrm{R}} \mathrm{A}$

(67) Uso inadecuado de la notación, escriben los conjuntos con letras minúsculas

(68) Uso inadecuado de la notación, escriben los elementos con letras mayúsculas

(69) Uso inadecuado de la notación, repiten un mismo elemento dentro del conjunto

Volumen $22 N^{\circ} 3$ Septiembre - Diciembre 2018. ISSN: 2244-7296 
CATÁLOGO DE ERRORES ASOCIADOS AL APRENDIZAJE DE LA TEORIA DE CONJUNTOS

Mariela Lilibeth Herrera y Celsa Álvarez

Pp 5-27

(70) Uso inadecuado de la notación, al definir el conjunto por comprensión no identifican al conjunto universal

(71) Uso inadecuado de la notación, para denotar un conjunto por extensión escriben el conjunto referencial y luego escriben la variable seguido del signo igual y mencionan los elementos

(72) Uso inadecuado de la notación, escribieron al conjunto vacío entre llaves

(73) Uso inadecuado de la notación, juntan las características de un conjunto escrito por comprensión y por extensión

(74) Uso inadecuado de la notación, escriben $\mathrm{a} \leq \mathrm{x} \geq \mathrm{b}$

(75) Uso inadecuado de la notación, se les olvida la variable, escriben $x \geq a \wedge \leq b$,

(76) Uso inadecuado de la notación, emplean a $\mathrm{x}$ como elemento genérico y a $\mathrm{P}(\mathrm{x})$ para expresar la condición que deben cumplir los elementos del conjunto

(77) Uso inadecuado de la notación, igualan el conjunto a su cardinal, para decir que $\mathrm{P}(\mathrm{B})$ tiene cuatro elementos escriben $\mathrm{P}(\mathrm{B})=4$.

(78) Uso inadecuado de la notación, a pesar de que "y" no aparece como parte del elemento genérico es parte del criterio de pertenencia

(79) Uso inadecuado de la notación, emplean dos variables separadas por coma como elemento genérico y el cuantificador universal delante de ellas, anteponen $\mathrm{P}(\mathrm{x})=$ al criterio de pertenencia

(80) Uso inadecuado de la notación, el elemento genérico aparece dos veces, primero solo y luego relacionado con el conjunto universal

(81) Uso incorrecto de la lógica, no emplean el símbolo de la implicación para conectar un razonamiento con el siguiente razonamiento

(82) Uso incorrecto de la lógica, en lugar de la conjunción colocan una coma

(83) Uso incorrecto de la lógica, no usan signos de agrupación

(84) Uso incorrecto de la lógica, no especifican las leyes de la lógica usada

(85) Uso incorrecto de la lógica, no emplean los paréntesis como signos de agrupación

(86) Uso incorrecto de la lógica, emplean la ley de simplificación de la disyunción

(87) Uso incorrecto de la lógica, no emplean la idempotencia de la conjunción y en su lugar hablan de una adjunción de la conjunción

(88) Uso incorrecto de la lóca, sólo consideran la implicación directa del bicondicional

(89) Uso incorrecto de la lógica, después de la conjunción no escriben el elemento genérico $(\mathrm{x} \in \mathrm{A} \wedge \in \mathrm{C})$

(90) Uso incorrecto de la lógica, no escriben correctamente el reciproco de una implicación

(91) Uso incorrecto de la lógica, escriben la negación del reciproco como contraejemplo

(92) Uso incorrecto de la lógica, para escribir el reciproco de una implicación sólo escriben la tesis y no colocan el símbolo de la implicación

(93) Uso incorrecto de la lógica, para escribir el reciproco de una implicación niegan la conjunción dada en la hipótesis 


\section{CATÁLOGO DE ERRORES ASOCIADOS AL APRENDIZAJE DE LA TEORIA DE CONJUNTOS}

Mariela Lilibeth Herrera y Celsa Álvarez

Pp 5-27

(94) Uso incorrecto de la lógica, para escribir el reciproco de una implicación sólo consideran una condición en la tesis del reciproco, dada una conjunción en la hipótesis

(95) Uso incorrecto de la lógica, emplean la transitividad de la implicación

\section{CONCLUSIÓN}

Los errores de nuestros estudiantes, muchas veces son producto de una distorsión de los canales de comunicación, de lo que los estudiantes creen escuchar o interpretan de lo que los docentes decimos en clase, que además, se ve reflejado en las notas que llevan. Cuando se lee lo que un estudiante escribe durante la clase de matemática, a veces parece que la comunicación no fue efectiva, el estudiante escribe cosas que el docente no dijo ni escribió, sin embargo, eso qué refleja, lo que el estudiante entendió, correcto o incorrecto, eso es lo que escribirá posteriormente en las evaluaciones. En este artículo, se sintetizan los errores cometidos por los estudiantes en las evaluaciones correspondientes a la teoría de conjunto, sin embargo, sería importante, recabar la información a través de los cuadernos de clases y evidenciar la perspectiva de los estudiantes, porque no podemos olvidar que una de las causas de los errores establecidas por Socas (1997) son los obstáculos didácticos, tampoco es tema de este artículo, explicar las causas de los errores. A pesar que no intentamos categorizar los errores, y que además, existen diversas taxonomías para clasificar los errores en el aprendizaje de la matemática, es importante, resaltar el hecho que comenzaron a emerger las siguientes categorías: (1) uso incorrecto de la lógica, (2) uso inadecuado de la notación algebraica, y (3) transformación defectuosa de la simbología algebraica del catálogo de los errores que tratamos de describir en este artículo 


\section{CATÁLOGO DE ERRORES ASOCIADOS AL APRENDIZAJE DE LA TEORIA DE CONJUNTOS}

Mariela Lilibeth Herrera y Celsa Álvarez

Pp 5-27

\section{REFERENCIAS}

Bachelard, G. (2007). La formación del Espíritu Científico. México: Siglo XXI Editores.

Balestrini, M. (2002). Cómo se Elabora un Proyecto de Investigación. Caracas: Consultores Privados, servicio Editorial.

Diccionario esencial de la Lengua Española (2006). España: Espasa Calpe

Ferrater, J. (2004). Diccionario de Filosofía. Tomo II (E-J). Barcelona: Ariel

Herrera, M., Gonzalez, A. (2011). Obstáculos y errores en el aprendizaje de los números irracionales. Trabajo de Maestría. Universidad de Carabobo. [Documento en línea]. Disponible en:

http://produccion-uc.bc.uc.edu.ve/documentos/trabajos/70002D93.pdf [Consulta: 2017, Mayo 25]

Lankshear, C. y Knobel, M. (2000). Problemas asociados con la metodología de la investigación cualitativa. PerfilesEducativos, XXII (87), 6-27

Pessoa, A. (1997). Cambios didácticos como consecuencia de las innovaciones curriculares. En UNESCO (Ed.). Proyecto Principal de Educación en América Latina y el Caribe. Boletín No. 44. Santiago. pp.7-15.

Piaget, J. (1978). Génesis del número en el niño.Buenos Aire: Editorial Guadalupe.

Rico, L., Castro, E. (1994). Errores y dificultades en el desarrollo del pensamiento numérico. Documento no publicado. Granada: Universidad de Granada. [Documento en línea]. Disponible en:

http://cumbia.ath.cx:591/pna/Archivos/RicoL94-148.PDF [Consulta: 2008, Junio 5]

Rico, L. (1995). Errores y dificultades en el aprendizaje de las matemáticas. En Kilpatrick, J., Rico, L., Gómez, P. (Eds.), Educación Matemática. Errores y dificultades de los estudiantes. Resolución de problemas. Evaluación. Historia (pp. 69-108). Bogotá: una empresa docente. [Documento en línea]. Disponible en: http://cumbia.ath.cx:591/pna/Archivos/RicoL94-148.PDF [Consulta: 2011, Junio 5]

Rodríguez, C., Gil, J, y García, E. (1996). Metodología de la Investigación Cualitativa. Málaga: Ediciones Aljibe 


\section{CATÁLOGO DE ERRORES ASOCIADOS AL APRENDIZAJE DE LA TEORIA DE CONJUNTOS}

\section{Mariela Lilibeth Herrera y Celsa Álvarez}

$\operatorname{Pp} 5-27$

Socas, M. (1997). Dificultades, obstáculos y errores en el aprendizaje de las matematicas en la Educación Secundaria. (En) Rico, L. (1997). La educación matemática en la enseñanza secundaria. Barcelona: ICE/Horsori pp.124-154

Zile y Nuñez (2008). Álgebra I. Valencia: Universidad de Carabobo 